\title{
Efficient Heuristic Algorithm for Scheduling Two-Stage Hybrid Flowshop with Sequence-Dependent Setup Times
}

\author{
Geun-Cheol Lee, ${ }^{1}$ Jung Man Hong, ${ }^{2}$ and Seong-Hoon Choi ${ }^{3}$ \\ ${ }^{1}$ College of Business Administration, Konkuk University, 120 Neungdong-ro, Gwangjin-gu, Seoul 143-701, Republic of Korea \\ ${ }^{2}$ LG CNS, FKI Tower, 24 Yeoui-daero, Yeongdeungpo-gu, Seoul 150-881, Republic of Korea \\ ${ }^{3}$ Department of Management Engineering, Sangmyung University, 31 Sangmyungdae-gil, Dongnam-gu, Cheonan, \\ Chungnam 330-720, Republic of Korea
}

Correspondence should be addressed to Seong-Hoon Choi; shchoi@smu.ac.kr

Received 29 January 2015; Revised 30 April 2015; Accepted 30 April 2015

Academic Editor: Yunqiang Yin

Copyright (C) 2015 Geun-Cheol Lee et al. This is an open access article distributed under the Creative Commons Attribution License, which permits unrestricted use, distribution, and reproduction in any medium, provided the original work is properly cited.

\begin{abstract}
We propose an efficient heuristic algorithm for two-stage hybrid flowshop scheduling with sequence-dependent setup times. In the past, metaheuristic approaches, which usually need long time, have been mostly used for the problem. In this study, due to practical reasons of the application that we consider, we need to obtain the solution of the problem within a reasonably short computational time, even for large-sized problems. In this study, we devise the proposed algorithm as a hybrid of two methods, that is, the beam search and NEH method, and we compare the performances with other existing local search methods. The results of the computational experiments show that the proposed algorithm solves the problems in a relatively shorter computation time, while the scheduling performances are superior to the existing methods.
\end{abstract}

\section{Introduction}

For the past several decades, many researchers have studied hybrid flowshop (HFS) scheduling problems. Among the HFS problems, two-stage HFS scheduling problems are considered simpler problems; however, there have been many studies considering two-stage HFS due to its practical and diverse applications [1]. In addition, if the HFS scheduling problems have sequence-dependent setup times (SDSTs), such problems can be more frequently seen in many real industries [2]. Thus, studies dealing with HFS scheduling problems with both two-stage and SDST, such as those by Lin and Liao [3] and Rossi et al. [4], usually have the actual applications for the problems. This study has the same characteristics as those in these literatures, which means that we consider the two-stage HFS scheduling problem with SDST and the considered problem has real applications occurring in three-dimensional (3D) automated optical inspection (AOI) machine operations.

HFS scheduling has been known as a complex problem because the simplest system in HFS, that is, two-stage HFS without SDST, was proven as NP-hard by Gupta [5] decades ago. Therefore, many researchers have developed heuristic methods to solve HFS scheduling problems regardless of the number of stages and the existence of setup times. In studies mentioned previously in this paper, which dealt with the two-stage HFS scheduling problems with SDST, heuristic methods were proposed $[3,4]$. For multistage HFS scheduling problems, most researchers used metaheuristic approaches, such as genetic algorithms, simulated annealing (SA), and immune algorithms (IAs). One of the most well-known studies was conducted by Kurz and Askin [6]. They considered the multistage HFS scheduling problem with SDST and proposed a genetic algorithm, the Random Keys Genetic Algorithm (RKGA), which has been the standard benchmark since then for other studies regarding the multistage HFS scheduling problem with SDST. Zandieh et al. [7] proposed an IA that performed better than the RKGA in large-sized problems; however, the computational times of the IA are also greater than the RKGA. Naderi et al. [8] proposed a hybrid simulated annealing (HSA) algorithm and compared it with the RKGA and the IA. The test results showed the outperformance of the HSA over the RKGA and the IA, with a slight increase of average computational time. Recently, Mirsanei et al. [2] 


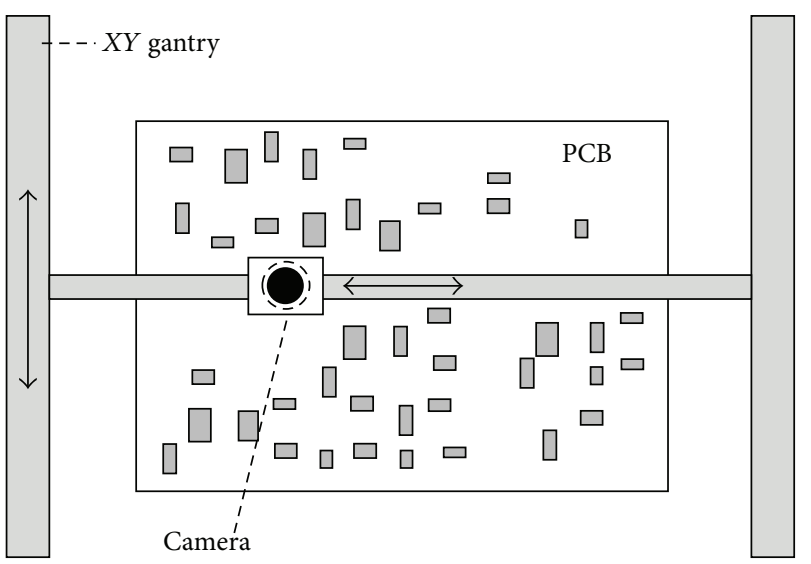

Figure 1: Structure of AOI machine [9].

proposed an SA algorithm that outperformed the RKGA and the IA with less computational time.

In this study, we consider the two-stage HFS scheduling problem with SDSTs, which originates from the real application, that is, automated optimal inspection (AOI) machine operations. The following section presents a detailed description of the AOI machine operation problem. Additionally, in this study, we consider important factors for operators in the practical fields, that is, that the AOI machine operations should be done within a reasonably short time, even for large operations. Thus, we propose a method that can solve the problem with up to 400 jobs within a very short time. (Note that problems with around 100 jobs were the largest in previous research.) To achieve such practical requirements, we devise the proposed method with two efficient heuristics other than metaheuristics, which commonly require long computation times. We combine two heuristics properly so that the proposed method is effective as well as efficient in solving the considered problems.

The rest of the paper is organized as follows. In the following section, we describe in detail the problem considered in this study. Section 3 presents the proposed method and Section 4 introduces the benchmark methods. In Section 5, we present the contents and results of the computational experiments for performance validation. We conclude the paper and mention future extensions of the study in the last section.

\section{Problem Description}

2.1. Three-Dimensional Automated Optical Inspection Machine Path Planning Problem (3D-AOI-PPP). In the printed circuit board (PCB) manufacturing process, AOI machines inspect whether or not the components on the $\mathrm{PCB}$ are correctly mounted with vision technologies. A typical aspect of the AOI machine focuses on camera movement, depicted in Figure 1, which is presented in Park et al. [9].

As we can see in Figure 1, the camera moves around over the PCB and acquires the images of the components mounted on the PCB; then, the CPU cores in the AOI machine perform the inspection by processing the images. One constraint is

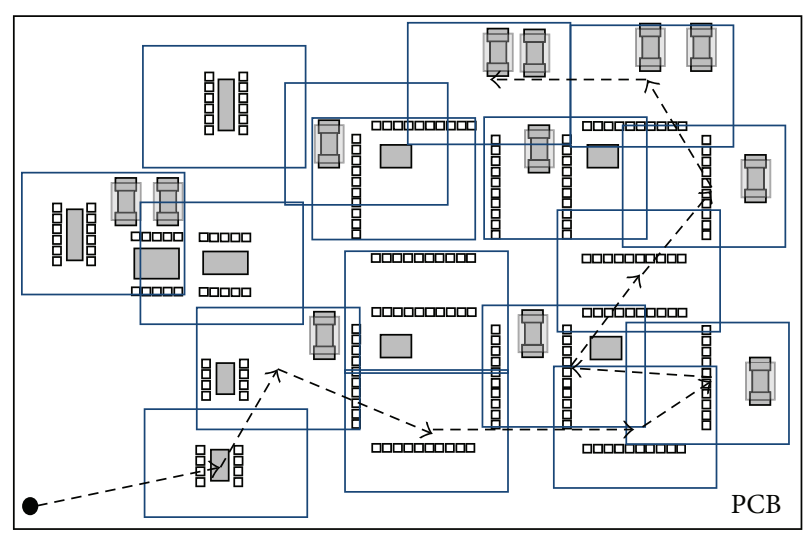

Figure 2: Camera path and FOVs on a PCB [9].

that the whole aspect of a PCB cannot be accommodated in a single camera shot. Thus, inspecting one PCB requires multiple camera shootings. Figure 2 shows the camera path on a PCB capturing multiple pictures at an AOI machine.

In Figure 2, the dotted lines and arrows form a camera path that shows the camera movements over the PCB. As Figure 2 shows, the camera moves from the center of a rectangular area to the center of another rectangular area, and so on. These rectangular areas are called fields of view (FOVs), which is the maximum region that can be acquired by one camera shoot. Each FOV contains a certain amount of mounted components on the PCB. To inspect the whole $\mathrm{PCB}$, the AOI machine needs to take pictures of all FOVs on the PCB.

The traditional AOI machine path planning problem is to find the optimal camera path that minimizes total working time [9]. Finding a solution to the problem is similar to finding the shortest camera path that must visit all FOVs; thus, the solution can be obtained by using TSP-based methods. In this study, the considered system is the threedimensional (3D) AOI machine, which uses 3D images when inspecting the correctness of component mountings.

Using 3D images enhances the quality of the inspection; however, the time of inspection increases due to $3 \mathrm{D}$ image processing. To reduce the image processing time, 3D AOI machines usually have multiple CPU cores. In traditional AOI machines, that is, $2 \mathrm{D}$ AOI machines, the total working time is mainly determined by the move time of the camera, whereas, in 3D AOI machines, the total working time is affected not only by the move time but also by the image processing times of FOV.

In this study, we consider the path planning problems on 3D AOI machines, in which the camera path, that is, the FOV visit sequence, is determined with the objective of minimizing the total working time. The following constraints should be met: the camera must visit the center positions of all given FOVs; when the picture of an FOV is acquired by the camera's shooting, the camera starts to move to the next FOV and the image processing of the FOV is simultaneously performed on one of the available CPU cores. If there is no 
TABLE 1: Comparison between 3D-AOI-PPP and 2S-HFS-SDST-SP.

\begin{tabular}{lcc}
\hline & 3D-AOI-PPP & 2S-HFS-SDST-SP \\
\hline & FOVs to be inspected & Jobs to be done \\
Problem configurations & One camera & Single machine at Stage 1 \\
& Multiple CPU cores & Shooting time \\
& Image processing time & Identical parallel machines at Stage 2 \\
& Processing time at Stage 1 & Processing time at Stage 2 \\
\hline Decision variable & Visit sequence of all FOVs & Sequence-dependent setup time between two jobs \\
\hline Objective functions & Minimize total working time & Operation sequence of all jobs \\
\hline
\end{tabular}

available CPU core, the image processing waits until a CPU becomes available.

\subsection{Two-Stage Hybrid Flowshop with Sequence-Dependent} Setup Time Scheduling Problem (2S HFS SDST SP). In this subsection, we first present that the considered problem, that is, the $3 \mathrm{D}-\mathrm{AOI}-\mathrm{PPP}$, is equivalent to a scheduling problem, which is the two-stage HFS with an SDST scheduling problem (2S-HFS-SDST-SP).

In 3D-AOI-PPP, inspection of one FOV consists of two operations: acquiring a picture of the FOV and processing a $3 \mathrm{D}$ image of the acquired FOV, which can be corresponded to the first and second operations in 2S-HFS-SDST-SP, respectively. An AOI machine has one camera and multiple CPU cores, which correspond to a single machine at Stage 1 and identical parallel machines at Stage 2 in 2S-HFS-SDST-SP. To inspect the next FOV in 3D-AOI-PPP, a certain amount of time is needed to move the camera, which can be considered as the SDST between two consecutively operated jobs at Stage 1 in the 2S-HFS-SDST-SP. In 3D-AOI-PPP, we decide the visit sequence of all the given FOVs to minimize the total working time, which is the same as with 2S-HFS-SDST-SP such that the job sequence is decided with the objective of minimizing the makespan. Consequently, the 3D-AOI-PPP can be considered the same problem as the 2S-HFS-SDSTSP. This is similar to the fact that the TSP is equivalent to a single machine with an SDST scheduling problem [10]. Table 1 summarizes the equivalencies between the 3D-AOI-PPP and the 2S-HFS-SDST-SP.

As mentioned previously, the two-stage (HFS) scheduling problem, even without the SDST, is NP-hard and there is no research that tries to solve the 2S-HFS-SDST-SP with mathematical models. However, mathematical formulation can be a tool to understand the problem precisely. In this study, using the formulation by Kurz and Askin [6], which presents mathematical formulation for the multistage HFS with an SDST problem, we present the mathematical model for the considered problem. First, we introduce the notation used in the formulation in the Notations.

Using Notations, we can present a mathematical model for the considered scheduling problem. In the formulation, we use job 0 as a dummy job to impose the setup time for job 1 and the processing time of job 0 is set to be zero. We also assume that all jobs must visit both stages according to the requirements of the AOI path planning problem.

Formulation. Consider the following:

$$
\begin{aligned}
& \text { Min } z \\
& \text { s.t. } \quad \sum_{j=1}^{N} x_{0 j 1}=1 \\
& \sum_{j=1}^{N} x_{0 j 2}=R \\
& \sum_{j=1}^{N} x_{i j k}=1, \quad \text { for } i=1,2, \ldots, N, k=1,2 \\
& \sum_{i=0}^{N} x_{i j k}=1, \quad \text { for } j=1,2, \ldots, N, k=1,2 \\
& c_{j 1}-c_{i 1}+\operatorname{BigM}\left(1-x_{i j 1}\right) \geq s_{i j}+p_{j 1}, \\
& \text { for } i=0,1, \ldots, N, j=1,2, \ldots, N \\
& c_{j 2}-c_{i 2}+\operatorname{BigM}\left(1-x_{i j 2}\right) \geq p_{j 2}, \\
& \text { for } i=0,1, \ldots, N, j=1,2, \ldots, N \\
& c_{j 2}-c_{j 1} \geq p_{j 2}, \quad \text { for } j=1,2, \ldots, N \\
& c_{j k} \geq c_{0 k}, \quad \text { for } j=1,2, \ldots, N, k=1,2 \\
& z \geq c_{j 2}, \quad \text { for } j=1,2, \ldots, N \\
& x_{i j k} \in\{0,1\}, \quad \forall i, j, k \\
& x_{i j k}=0, \quad \forall i=j, k=1,2 \\
& c_{j k} \geq 0, \quad \text { for } j=0,1, \ldots, N, k=1,2 \text {. }
\end{aligned}
$$

Equation (1) shows the objective function of the problem, that is, minimizing makespan. Constraints (2) and (3) ensure that the jobs are scheduled on a machine at Stage 1 and on $R$ machines at Stage 2, respectively. Constraints (4) and (5) ensure that each job is sequenced immediately before and 
after only one job, respectively, on only one machine at each stage. Constraint (6) ensures that the completion time of job $j$ at Stage 1 is greater than that of job $i$ by at least the processing time of job $j$ at Stage 1 plus the setup time between jobs $i$ and $j$, if job $i$ is sequenced immediately before job $j$ at Stage 1 . In the equation, BigM is specified as a very large value, ensuring the inequality is always met. Constraint (7) ensures that the completion time of job $j$ at Stage 2 is greater than that of job $i$ by at least the processing time of job $j$ at Stage 2, if job $i$ is sequenced immediately before job $j$ on a machine at Stage 2 . Constraint (8) ensures that the completion time of a job at Stage 2 is always greater than that of the same job at Stage 1 by at least the processing time of the job at Stage 2. Constraint (9) forces job 0 to be sequenced first. Constraint (10) links the objective function and the decision variables. Constraints (11), (12), and (13) impose the boundary of the decision variables. Note that the formulation is introduced to describe the considered scheduling problem in more detail. Solving the problem with the mathematical model is not appropriate for this study because, for practical reasons, we need to solve a relatively large-sized problem and such problems need to be solved within a reasonably short time.

\section{Proposed Methods}

To solve the considered problem, we propose a method that combines two heuristics, that is, beam search and NEH. The beam search introduced by Ow and Morton [11] in scheduling is a truncated branch and bound search, where only a small number of solutions are maintained in each level of the search tree. $\mathrm{NEH}$ is a well-known and effective flowshop scheduling heuristic proposed by Nawaz et al. [12]. An NEHbased heuristic for the problem was proposed by Lee et al. [13], and the solutions obtained by this heuristic were effective and efficient. In this study, we think that embedding the $\mathrm{NEH}$ procedure in the beam search enables a more diverse search on the solution space. Although well-known metaheuristics could search even wider solution space and find better solutions than the proposed method, metaheuristics will need much more computation time, which are not acceptable in the considered problem due to the practical reason. In this study, with the proposed beam search method, we could obtain the solutions more effectively as well as efficiently.

The proposed method consists of three parts: the first part is constructing the initial solution, the second part is the beam search with the embedded $\mathrm{NEH}$, and the last part is the improvement procedure by job-pair interchange and job insertion, which is also modified as a beam search style. In the second part, we present the procedure of constructing the initial solution. We based the procedure on the famous Johnson's rule, which was originally used for a two-stage flowshop scheduling problem with minimizing makespan [14]. The computational complexity of Johnson's rule is known as $O(N \log N)$ [15]. In the considered problem, we assume the processing times of all jobs at Stage 1 to be the same because they are the shooting times, which are the same regardless of FOVs. Thus, Johnson's rule can be applied by sorting the jobs in descending order of the processing times at
Stage 2. The detailed procedure can be described as follows. In the procedure, $S$ denotes the sequence of ordered jobs, and $S_{x}$ denotes the job at position $x$ in $S$. Thus, $S=\left\{S_{1}-S_{2}-\cdots-S_{N}\right\}$, and $U$ denotes the set of jobs whose sequence is not decided.

\subsection{Johnson's Rule-Based Procedure (JS)}

Step $0 . U:=\{1,2, \ldots, N\}, x:=1$.

Step 1. $S_{x}:=\arg \max _{i \in U}\left\{p_{i 2}\right\} . U:=U-\left\{S_{x}\right\} . x:=x+1$.

Step 2. If $x \leq N$, go to Step 1; otherwise, $S$ is the final solution. STOP.

After obtaining the initial solution with the above JS, we apply the beam search with the embedded NEH to improve the solution. While the NEH maintains only one best solution at each level for branching, the beam search proposed in this study maintains multiple best solutions. The maximum number of solutions maintained at each level of the search tree is called beam width, which is denoted as $w$ in the remaining of the paper. Because the $\mathrm{NEH}$ evaluates a total of $\{N(N+1) / 2\}-1$ sequences [16] and the proposed beam search evaluates $w$-fold number of sequences compared to the $\mathrm{NEH}$, the total number of sequences evaluated in the beam search is $w[\{N(N+1) / 2\}-1]$. Since $w$ is a constant, we obtain a computational complexity of $O\left(N^{3}\right)$. We present the detailed procedure of the beam search in the following procedure. Let $S^{w}$ be the job sequence of the $w$ th best solution and let $S_{x}^{w}$ denote the job at position $x$ in $S^{w}$. Thus, $S^{w}=$ $\left\{S_{1}^{w}-S_{2}^{w}-\cdots-S_{N}^{w}\right\}$.

\subsection{Beam Search Procedure (BS $(w))$}

Step 0 . Let $S$ be the solution obtained by JS. Set $S^{w}:=S$, for all $w . U:=\{1,2, \ldots, N\}-\left\{S_{1}\right\} . x:=2$.

Step 1. If $U=\emptyset$, go to Step 4; otherwise, select job $S_{x}$.

Step 2. Generate candidate solutions by putting job $S_{x}$ at every available position in $S^{w}$ for all $w$ and obtain the makespans of the candidate solutions.

Step 3. Update $S^{w}$ with the $w$ best solutions in Step 2. $U:=$ $U-\left\{S_{x}\right\} . x:=x+1$. Go to Step 1 .

Step $4 . S^{1}$ is the final solution. STOP.

In Step 2, the available positions for job $S_{x}$ are 1st, 2nd,..., $x$ th positions in $S^{w}$. For example, job $S_{2}$ has two available positions, whereas job $S_{N}$ has $N$ available positions, resulting in $N$ candidate solutions. In the Appendix, illustrations of solving an example problem by the beam search procedure as well as Johnson's rule-based procedure are presented.

Because the beam search maintains $w$ best solutions at each level of its search tree, we would expect a wider solution space search than the NEH. However, the improvement of the solution quality by the beam search presented previously is not sufficient and there is room for enhancement. Therefore, 
we propose two improvement procedures: one is pairwise interchange and the other is single job insertion, and we devised both procedures to contain the beam search concept. In the rest of the paper, the former improvement procedure is denoted as BPI (beam search pairwise interchange) and the latter is denoted as BNI (beam search NEH insertion). We present the following two procedures, respectively. In the procedure, $I$ denotes the maximum number of iterations, which is a prespecified value.

\subsection{Procedure of $B P I(w, I)$}

Step $0($ Iteration $:=0)$. Let $S$ be a given solution. Set $S^{w}:=S$, for all $w$.

Step 1. Do the pairwise interchange for all $S^{w}$.

Step 1.1. Schedule the jobs with $S^{w}$ on the two-stage HFS.

Step 1.2. Select a job arbitrarily among the jobs on the machine with the largest load at Stage 2.

Step 1.3. Select another job arbitrarily on machines except the machine from which a job has already been selected in Step 1.2 .

Step 1.4. Generate the modified sequence by changing the positions of the two selected jobs. If the makespan of the modified sequence decreases, update $S^{w}$ with the modified sequence.

Step 2. Update $S^{w}$ with the $w$ best solutions in Step 1.

Step 3. If Iteration $=I$, then $S^{1}$ is the final solution. STOP; $/ \mathrm{w}$, Iteration $:=$ Iteration +1 , go to Step 1 .

\subsection{Procedure of $B N I(w, I)$}

Step 0 (Iteration $:=0)$. Let $S$ be a given solution. Set $S^{w}:=S$, for all $w$.

Step 1. Do the NEH interchange for all $S^{w}$.

Step 1.1. Select a job arbitrarily.

Step 1.2. Generate candidate solutions by putting the selected job at every position in $S^{w}$ and obtain the makespans of the candidate solutions.

Step 2. Update $S^{w}$ with the $w$ best solutions in Step 1.

Step 3. If Iteration $=I$, then $S^{1}$ is the final solution. STOP; $\mathrm{o} / \mathrm{w}$, Iteration $:=$ Iteration +1 , go to Step 1 .

In both procedures, Step 0 needs a given solution to initiate the procedure, which can be obtained from JS or BS or even BPI or BNI. As mentioned previously, the proposed method consists of the three phases: the initial solution is first obtained using JS; then, BS is applied, and the final improvement procedure is applied with a combination of BPI and BNI. In this study, we use BPI and BNI alternately with small iterations. For example, the procedures are not applied with "JS $\rightarrow \mathrm{BS}(2) \rightarrow \mathrm{BPI}(2,100) \rightarrow \mathrm{BNI}(2,10)$ "; rather they are applied with "JS $\rightarrow \mathrm{BS}(2) \rightarrow \mathrm{BPI}(2,50) \rightarrow \mathrm{BNI}(2$, $5) \rightarrow \operatorname{BPI}(2,50) \rightarrow \operatorname{BNI}(2,5)$ ”. We determine an alternating number of BPI and BNI through preliminary tests, and the parameter values needed in the procedure, that is, $w, I$, are also set through the preliminary tests.

After the preliminary tests, the following procedure is selected as the final proposed method, that is, "JS $\rightarrow$ $\mathrm{BS}(2) \rightarrow \mathrm{BNI}(2,400) \rightarrow \mathrm{BPI}(2,4000) \rightarrow \mathrm{BNI}(2,400) \rightarrow$ $\mathrm{BPI}(2,4000) \rightarrow \mathrm{BNI}(2,400) \rightarrow \mathrm{BPI}(2,4000) \rightarrow \mathrm{BNI}(2$, $400) \rightarrow \operatorname{BPI}(2,4000)$ ". There could be several comments about the procedure. First, the beam width value is set to two in all BS, BNI, and BPI. We tested other beam width values, such as three and four, which gave similar performances but showed more computing times. When we alternate BNI and $\mathrm{BPI}$, we put BNI first and decided to repeat the alternation four times. The maximum iteration numbers are set to 400 and 4,000 in BNI and BPI, respectively. Note that one iteration in BNI requires more time than one iteration in BPI. All these configurations in the proposed method are decided by a series of parameter tuning work. We denote the proposed method as $\mathrm{BS}+$ in the rest of the paper.

\section{Benchmarks}

To investigate the performance of the proposed algorithm, we introduce three benchmark methods, including two metaheuristics and one existing heuristic method. In this section, we present each benchmark method.

4.1. Genetic Algorithm (GA). For the first benchmark method, we introduce a genetic algorithm that borrows the procedure of the RKGA, which is the algorithm proposed by Kurz and Askin [6], to solve the problem of scheduling HFS with SDST. We modify the introduced genetic algorithm for the problem; that is, the solution representation no longer uses the random keys, and, rather, we use the permutation sequence as the solution representation for the GA. However, many other features in the genetic algorithms are similar to those in RKGA; the population size is set to 100; that is, each generation has 100 chromosomes. The first generation of the first chromosome is constructed by using JS, and the remaining 99 chromosomes are obtained by applying a single pairwise interchange on the first chromosome.

In each iteration, the next generation is constructed as follows: $20 \%$ of the sequences (i.e., chromosomes) in the parent generation, which have the minimum makespans, are automatically moved to the next generation; $1 \%$ of chromosomes in the next generation are randomly generated. We obtain the remaining $79 \%$ of chromosomes in the next generation by applying crossover operations on the parent generation; that is, making a new chromosome requires that we randomly select two different chromosomes (denoted as 1st and 2nd here) in the parent generation. Each gene value of the new chromosome is then decided as a corresponding gene of the 1 st or 2 nd chromosome. For each gene, a random number is generated. If the value is less than 0.7 , the gene value of the 1st chromosome is used; otherwise, the gene value of the $2 \mathrm{nd}$ 
chromosome is used. Through this crossover procedure, we construct $79 \%$ of the next generation. The iteration continues until finding 2,000 consecutive generations without solution improvement. This genetic algorithm is denoted as GA in the rest of the paper.

4.2. Simulated Annealing (SA). In addition to the previously introduced GA, another metaheuristic method, that is, simulated annealing (SA), is additionally used as a benchmark method. SA has been one of the most widely used metaheuristic methods in combinatorial optimization problems since being introduced by Kirkpatrick et al. [17]. The performance of this method is mainly dependent on a neighborhood search scheme, that is, the procedure of finding good solutions near the current solution.

In this study, several features of the SA are manipulated for the considered problem. Once the initial solution is generated by the JS, in each iteration (i.e., at one temperature), a new solution is generated through a neighborhood search. In this study, positions of two randomly selected jobs in the current solution are interchanged to generate a neighborhood solution. This process repeats until the number of neighborhood solutions reaches the prespecified neighborhood size. If the best among the neighborhood solutions has a shorter makespan than the current solution, the current solution moves to the new solution. Otherwise, the current solution does not change; however, with very small amount of the prespecified probability, the solution moves to the new solution, even if the solution quality is not improved. This feature makes the SA more powerful; that is, it enables the search to escape from the local optimum.

To specify the search characteristics and termination condition of the SA, we used parameters introduced in Johnson et al. [18]. First, TempFactor is a multiplier for the current temperature, which specifies the decrease in the temperature in each iteration. The neighborhood size can be determined by SizeFactor, by multiplying its value with the job size. Finally, the SA procedure terminates if the acceptance ratio in the past five iterations is less than the MinPercent value. In this study, we set the values of TempFactor, SizeFactor, and MinPercent to $0.98,10$, and $1 \%$, respectively.

4.3. NEH-Based Heuristic. The last benchmark method is the heuristic method proposed by Lee et al. [13], which was developed for solving the same scheduling problem considered in this study. Actually, this benchmark method is the same as the proposed method, if the beam width of the proposed method is set to be one. This approach consists of the following four parts performed sequentially; first (1) using JS, the initial solution is constructed, then (2) classic NEH, (3) the pairwise interchange, and (4) NEH insertion are applied to the solution respectively for the improvements. We denote this method as $\mathrm{NEH}+$ in the rest of the paper.

\section{Computational Experiments}

To verify the performance of the proposed method, we conducted computation experiments. In these computational experiments, we investigate the performance of the proposed
TABLE 2: Performance results of the tested methods across the different job sizes.

\begin{tabular}{lcccc}
\hline \# of jobs & GA & SA & NEH+ & BS+ \\
\hline \multirow{2}{*}{50} & $80.0^{\dagger}$ & 75.8 & 75.4 & 74.8 \\
& $(0)^{\ddagger}$ & $(7)$ & $(6)$ & $(17)$ \\
\hline \multirow{2}{*}{100} & 154.0 & 141.0 & 137.5 & 137.2 \\
& $(0)$ & $(2)$ & $(9)$ & $(19)$ \\
\hline \multirow{2}{*}{200} & 314.2 & 283.5 & 273.2 & 273.2 \\
& $(0)$ & $(3)$ & $(11)$ & $(16)$ \\
\hline \multirow{2}{*}{400} & 636.2 & 609.9 & 530.5 & 530.6 \\
& $(0)$ & $(1)$ & $(18)$ & $(11)$ \\
\hline \multirow{2}{*}{ Overall } & 296.1 & 277.5 & 254.1 & 253.9 \\
& $(0)$ & $(13)$ & $(44)$ & $(63)$ \\
\hline
\end{tabular}

${ }^{\dagger}$ Average makespan value.

${ }^{\ddagger}$ Number of instances that the corresponding method gave the best solution.

method compared to those of the benchmark methods introduced in the earlier section. In the following subsections, we present features of the tested problems used in the computational experiments, and we present the results of the experiments in scheduling and computing performances. All the tested methods were implemented in $C$ language and the computational experiments were performed on a PC with 3.20 GHz i5 CPU and 3 GB RAM.

5.1. Problem Instances. In this study, the tested problems are randomly generated; however, the ranges of values are set based on real 3D AOI problems. That is, we first generate the problem data for the 3D AOI machine path planning problem and transform it into data for the two-stage HFS with an SDST scheduling problem. For example, we first generate $x$-, $y$-coordinates of FOVs for 3D AOI machine path planning problem and use the coordinates to calculate the move times between the two FOVs considering the speed information of the camera; these data are the SDSTs in the scheduling problem. In addition, the $3 \mathrm{D}$ image processing time for each FOV in the AOI problem is the processing time of the corresponding job at Stage 2 in the scheduling problem, and so on.

To generate the various types of problems, we vary the number of jobs in four levels, that is, 50, 100, 200, and 400, and the number of machines at Stage 2 in three levels, that is, 4,8 , and 16 . For each instance of the problem, we generate ten replications. In total, $120(=4 \times 3 \times 10)$ problem instances are generated and used for the experiments. As you can see from the job sizes of the generated problems, in this study, we are focusing on rather large job-size problems, that is, mostly over 100 and up to 400, which are efforts reflecting the practical needs in operating the $3 \mathrm{D}$ AOI machines. From a scheduling perspective, they are also relatively large problems compared to the tested problems in the previous HFS scheduling research.

5.2. Test Results. In the test, we compare the proposed method (BS+) with three benchmarks, that is, GA, SA, and $\mathrm{NEH}+$ in terms of the scheduling performance and computing speed. Table 2 shows the objective function values, that 
TABLE 3: Performance results of the tested method across different numbers of machines.

\begin{tabular}{lcccc}
\hline \# of machines & GA & SA & NEH+ & BS+ \\
\hline \multirow{2}{*}{4} & $415.1^{\dagger}$ & 404.0 & 403.9 & 403.5 \\
& $(0)^{\ddagger}$ & $(10)$ & $(9)$ & $(21)$ \\
\hline \multirow{2}{*}{8} & 247.5 & 218.6 & 213.5 & 213.3 \\
& $(0)$ & $(3)$ & $(15)$ & $(22)$ \\
\hline \multirow{2}{*}{16} & 225.7 & 210.0 & 145.0 & 145.0 \\
& $(0)$ & $(0)$ & $(20)$ & $(20)$ \\
\multirow{2}{*}{ Overall } & 296.1 & 277.5 & 254.1 & 253.9 \\
& $(0)$ & $(13)$ & $(44)$ & $(63)$ \\
\hline
\end{tabular}

${ }^{\dagger}$ Average makespan value.

${ }^{\ddagger}$ Number of instances that the corresponding method gave the best solution.

is, makespans, obtained by all the tested methods across the different job sizes. In Table 2, values in parenthesis are the number of instances for which the corresponding method performed best.

As you can see from Table 2, NEH+ and BS+ outperform the two metaheuristic methods. Although SA performs relatively well in the small-sized problems, the proposed method dominates other methods in the problem for the job size of 50 . The proposed method, that is, $\mathrm{BS}+$, and $\mathrm{NEH}+$ show very close performance results in the makespan value. However, we see the superiority of the proposed method when we look at the values in parenthesis, that is, the number of instances the method gives the minimum makespan. The overall number is the biggest. Moreover, the number is consistently larger than others across the different job size problems.

Table 3 shows the scheduling performance results of the tested methods across the different number of machines at Stage 2. As the results of Table 2 show, the proposed method (BS+) and $\mathrm{NEH}+$ show better performance over the two metaheuristics, regardless of the number of machines. While NEH+ has slightly weaker results in the small number of machines, BS+ consistently performs better than others do across the different numbers of machines in terms of makespan values and number of best instances.

In the next results of the test, we show the computing speed of the tested method. Table 4 shows the computational times of the tested method across the job sizes in seconds. As you can see from Table 4, the proposed method shows the dominant performance in computing speed. Although SA performs better in a job size of 50, computational time of SA increases as the job size increases. Additionally, Table 5 shows that the proposed method obtains the solutions the fastest among the tested methods, regardless of the number of machines at Stage 2. In addition to the scheduling performance in the previous test results, we can argue that the proposed method gives effective and efficient solutions in the two-stage HFS scheduling problems.

Through the various results of the experiments, we can see that the proposed method shows the outperformance over the benchmarks in diverse problems. Although a slight scheduling performance gap between $\mathrm{NEH}+$ and the proposed
TABLE 4: Computational times of the tested method across different job sizes.

\begin{tabular}{lcccc}
\hline \# of jobs & GA & SA & NEH+ & BS+ \\
\hline \multirow{2}{*}{50} & $7.1^{\dagger}$ & 0.7 & 1.0 & 0.8 \\
& $(0)^{\ddagger}$ & $(21)$ & $(0)$ & $(9)$ \\
\hline \multirow{2}{*}{100} & 21.4 & 3.2 & 3.6 & 2.8 \\
& $(0)$ & $(3)$ & $(0)$ & $(27)$ \\
\hline \multirow{2}{*}{200} & 69.2 & 13.4 & 13.4 & 10.3 \\
& $(0)$ & $(0)$ & $(0)$ & $(30)$ \\
\hline \multirow{2}{*}{400} & 120.1 & 53.7 & 53.0 & 41.1 \\
& $(1)$ & $(0)$ & $(0)$ & $(28)$ \\
\hline \multirow{2}{*}{ Overall } & 54.5 & 17.7 & 17.7 & 13.8 \\
& $(1)$ & $(24)$ & $(0)$ & $(94)$
\end{tabular}

${ }^{\dagger}$ Average computational times in seconds.

${ }^{\ddagger}$ Number of instances that the corresponding method gave the solution the fastest.

TABLE 5: Computational times of tested methods across different numbers of machines.

\begin{tabular}{lcccc}
\hline \# of machines & GA & SA & NEH+ & BS+ \\
\hline \multirow{2}{*}{4} & $33.9^{\dagger}$ & 14.5 & 11.6 & 8.2 \\
& $(0)^{\ddagger}$ & $(3)$ & $(0)$ & $(37)$ \\
\hline \multirow{2}{*}{8} & 65.5 & 18.0 & 18.2 & 14.2 \\
& $(0)$ & $(8)$ & $(0)$ & $(32)$ \\
\hline \multirow{2}{*}{16} & 64.0 & 20.7 & 23.4 & 18.9 \\
& $(1)$ & $(13)$ & $(0)$ & $(25)$ \\
\multirow{2}{*}{ Overall } & 54.5 & 17.7 & 17.7 & 13.8 \\
& $(1)$ & $(24)$ & $(0)$ & $(94)$ \\
\hline
\end{tabular}

${ }^{\dagger}$ Average computational times in seconds.

${ }^{\ddagger}$ Number of instances that the corresponding method gave the solution the fastest.

method can be seen, BS+ definitely delivers a higher number of best instances as compared to NEH+. Furthermore, in terms of the computational time, we can say that $\mathrm{BS}+$ is more efficient than $\mathrm{NEH}+$.

\section{Conclusions}

In this study, we considered the two-stage HFS scheduling problem with SDSTs. The considered problem was motivated by the practical application from automated optimal inspection machine operations in which the jobs need to be done within a reasonably short time. Thus, we proposed an efficient heuristic algorithm embedded with the NEH procedure in the beam search framework. From the series of the computational experiments, we found that the proposed algorithm produced the solutions in a shorter time than the benchmark methods, including two metaheuristic methods. Furthermore, the experimental results also showed that the scheduling performance of the proposed algorithm is placed in the best among the benchmark methods. 
TABLE 6

\begin{tabular}{ccccccc}
\hline Job & 1 & 2 & 3 & 4 & 5 & 6 \\
\hline$p_{i 2}$ & 122 & 226 & 83 & 319 & 64 & 217 \\
\hline
\end{tabular}

TABLE 7

\begin{tabular}{lcccccc}
\hline$(i, j)$ & 1 & 2 & 3 & 4 & 5 & 6 \\
\hline 1 & 0 & 45 & 34 & 24 & 40 & 34 \\
2 & 45 & 0 & 56 & 64 & 81 & 56 \\
3 & 34 & 56 & 0 & 53 & 33 & 68 \\
4 & 24 & 64 & 53 & 0 & 44 & 25 \\
5 & 40 & 81 & 33 & 44 & 0 & 67 \\
6 & 34 & 56 & 68 & 25 & 67 & 0 \\
\hline
\end{tabular}

\section{Appendix}

\section{A. Solving an Example Problem}

\section{A.1. Example Problem Configurations}

Number of jobs $(N): 6$.

Number of machines at Stage $2(R): 2$.

Processing times of each job at Stage 1: 20.

Processing times of job $i$ at Stage 2 (see Table 6).

Sequence-dependent setup times between two jobs (see Table 7).

First, we illustrate the step-by-step procedure of solving the above example problem by Johnson's rule-based procedure (JS).

\section{A.2. Solving the Example by JS}

Step $0 . U:=\{1,2,3,4,5,6\}, x:=1$.

Step 1. $S_{1}:=\mathbf{4}=\arg \max _{i \in\{1,2,3,4,5,6\}}\left\{p_{i 2}\right\} . U:=\{1,2,3,5,6\}$ $(=U-\{4\}) \cdot x:=2(=x+1)$.

Step 2. $x(=2) \leq N(=6)$. Thus, go to Step 1 .

Step 1. $S_{2}:=\mathbf{2}=\arg \max _{i \in\{1,2,3,5,6\}}\left\{p_{i 2}\right\} . U:=\{1,3,5,6\}$. $x:=3$.

Step 2. $x(=3) \leq 6$. Thus, go to Step 1 .

Step 1. $S_{3}:=\mathbf{6}=\arg \max _{i \in\{1,3,5,6\}}\left\{p_{i 2}\right\} . U:=\{1,3,5\} . x:=4$.

Step 2. $x(=4) \leq 6$. Thus, go to Step 1 .

Step 1. $S_{4}:=\mathbf{1}=\arg \max _{i \in\{1,3,5\}}\left\{p_{i 2}\right\} . U:=\{3,5\} . x:=5$.

Step 2. $x(=5) \leq 6$. Thus, go to Step 1 .

Step $1 . S_{5}:=\mathbf{3}=\arg \max _{i \in\{3,5\}}\left\{p_{i 2}\right\} . U:=\{5\} . x:=6$.

Step 2. $x(=6) \leq 6$. Thus, go to Step 1 .

Step $1 . S_{6}:=\mathbf{5}=\arg \max _{i \in\{5\}}\left\{p_{i 2}\right\} . U:=\{\} . x:=7$.
TABLE 8

\begin{tabular}{ccc}
\hline$\#$ & Candidate solutions & Makespan \\
\hline 1 & $\{4-\underline{2}-6-1-3-5\}^{* *}$ & 623 \\
2 & $\{\underline{2}-4-6-1-3-5\}^{*}$ & 609
\end{tabular}

* Best solution among all candidate solutions in this step.

** Second best solution among all candidate solutions in this step.

TABLE 9

\begin{tabular}{|c|c|c|c|c|c|}
\hline \multicolumn{3}{|c|}{ For $S^{1}$} & \multicolumn{3}{|c|}{ For $S^{2}$} \\
\hline \# & $\begin{array}{c}\text { Candidate } \\
\text { solutions }\end{array}$ & Makespan & \# & $\begin{array}{c}\text { Candidate } \\
\text { solutions }\end{array}$ & Makespan \\
\hline 1 & $\{2-4-\underline{6}-1-3-5\}$ & 609 & 4 & $\{4-2-\underline{6}-1-3-5\}$ & 623 \\
\hline 2 & $\{2-\underline{6}-4-1-3-5\}^{* *}$ & 582 & 5 & $\{4-\underline{6}-2-1-3-5\}$ & 586 \\
\hline 3 & $\{\underline{6}-2-4-1-3-5\}$ & 591 & 6 & $\{\underline{6}-4-2-1-3-5\}^{*}$ & 580 \\
\hline
\end{tabular}

* Best solution among all candidate solutions in this step.

** Second best solution among all candidate solutions in this step.

Step 2. $x(=7)>$ 6. Thus, $S$, that is, $\{$ 4-2-6-1-3-5 $\}$ $\left(=\left\{S_{1}-S_{2}-S_{3}-S_{4}-S_{5}-S_{6}\right\}\right)$, is the final solution. STOP.

Using JS, we obtained a solution, $\{4-2-6-1-3-5\}$, with makespan value 623. Next, the step-by-step procedure of solving the same example by the proposed beam search procedure with beam width two, that is, BS(2), is presented.

\section{A.3. Solving the Example by BS(2)}

Step $0 . S:=\{4-2-6-1-3-5\}$ (=the solution obtained by JS). $S^{1}:=\{4-2-6-1-3-5\}$ and $S^{2}:=\{4-2-6-1-3-5\} . U:=\{1,2,3$, $5,6\}(=\{1,2,3,4,5,6\}-\{4\}) \cdot x:=2$.

Step $1(U \neq \emptyset)$. Thus, select $S_{2}$, that is, job 2 .

Step 2. For $S^{1}$ and $S^{2}$, there are two candidate solutions as shown in Table 8.

Step 3. $S^{1}:=\{2-4-6-1-3-5\}$, which is the best solution, and $S^{2}:=\{4-2-6-1-3-5\}$, which is the second best solution. $U:=$ $\{1,3,5,6\} . x:=3(=x+1)$. Go to Step 1 .

Step $1(U \neq \emptyset)$. Thus, select $S_{3}$, that is, job 6 .

Step 2. All candidate solutions are listed in Table 9.

Step 3. $S^{1}:=\{6-4-2-1-3-5\}$, which is the best solution, and $S^{2}:=\{2-6-4-1-3-5\}$, which is the second best solution. $U:=$ $\{1,3,5\} . x:=4$. Go to Step 1 .

Step $1(U \neq \emptyset)$. Thus, select $S_{4}$, that is, job 1 .

Step 2. All candidate solutions are listed in Table 10.

Step 3. $S^{1}:=\{2-1-6-4-3-5\}$, which is the best solution, and $S^{2}:=\{6-4-2-1-3-5\}$, which is the second best solution. $U:=$ $\{3,5\} . x:=5$. Go to Step 1 .

Step $1(U \neq \emptyset)$. Thus, select $S_{5}$, that is, job 3 . 
TABLE 10

\begin{tabular}{cccccc}
\hline & $\begin{array}{c}\text { For } S^{1} \\
\text { Candidate } \\
\text { solutions }\end{array}$ & Makespan & $\#$ & $\begin{array}{c}\text { For } S^{2} \\
\text { Candidate } \\
\text { solutions }\end{array}$ & Makespan \\
\hline 1 & $\{6-4-2-1-1-3-5\}^{* *}$ & $\mathbf{5 8 0}$ & 5 & $\{2-6-4-\underline{1}-3-5\}$ & 582 \\
2 & $\{6-4-\underline{1}-2-3-5\}$ & 585 & 6 & $\{2-6-1-4-3-5\}$ & 632 \\
3 & $\{6-\underline{1}-4-2-3-5\}$ & 623 & 7 & $\{2-\underline{1}-6-4-3-5\}^{*}$ & $\mathbf{5 7 1}$ \\
4 & $\{\underline{1}-6-4-2-3-5\}$ & 581 & 8 & $\{\underline{1}-2-6-4-3-5\}$ & 630 \\
\hline
\end{tabular}

${ }^{*}$ Best solution among all candidate solutions in this step.

${ }^{* *}$ Second best solution among all candidate solutions in this step.

TABLE 11

\begin{tabular}{|c|c|c|c|c|c|}
\hline \multicolumn{3}{|c|}{ For $S^{1}$} & \multicolumn{3}{|c|}{ For $S^{2}$} \\
\hline \# & $\begin{array}{l}\text { Candidate } \\
\text { solutions }\end{array}$ & Makespan & $\#$ & $\begin{array}{l}\text { Candidate } \\
\text { solutions }\end{array}$ & Makespan \\
\hline 1 & $\{2-1-6-4-\underline{3}-5\}^{*}$ & $\mathbf{5 7 1 ^ { \dagger }}$ & 6 & $\{6-4-2-1-\underline{3}-5\}$ & 580 \\
\hline 2 & $\{2-1-6-\underline{3}-4-5\}$ & 697 & 7 & $\{6-4-2-\underline{3}-1-5\}$ & 587 \\
\hline 3 & $\{2-1-3-6-4-5\}$ & 659 & 8 & $\{6-4-\underline{3}-2-1-5\}^{*}$ & $571^{\dagger}$ \\
\hline 4 & $\{2-3-1-6-4-5\}$ & 620 & 9 & $\{6-\underline{3}-4-2-1-5\}$ & 625 \\
\hline 5 & $\{3-2-1-6-4-5\}$ & 647 & 10 & $\{\underline{3}-6-4-2-1-5\}$ & 615 \\
\hline
\end{tabular}

${ }^{*}$ Best solution among all candidate solutions in this step.

${ }^{\dagger}$ Two different solutions have the same minimum makespan.

TABLE 12

\begin{tabular}{cccccc}
\hline & $\begin{array}{c}\text { For }^{1} \\
\text { \# } \\
\text { Candidate } \\
\text { solutions }\end{array}$ & Makespan & $\#$ & $\begin{array}{c}\text { For } S^{2} \\
\text { Candidate } \\
\text { solutions }\end{array}$ & Makespan \\
\hline 1 & $\{2-1-6-4-3-\underline{5}\}^{*}$ & $\mathbf{5 7 1}^{\ddagger}$ & 1 & $\{6-4-3-2-1-\underline{5}\}^{*}$ & $\mathbf{5 7 1}^{\ddagger}$ \\
2 & $\{2-1-6-4-5-3\}^{*}$ & $\mathbf{5 7 1}^{\ddagger}$ & 2 & $\{6-4-3-2-\underline{5}-1\}$ & 607 \\
3 & $\{2-1-6-\underline{5}-4-3\}$ & 677 & 3 & $\{6-4-3-\underline{5}-2-1\}$ & 647 \\
4 & $\{2-1-\underline{5}-6-4-3\}$ & 658 & 4 & $\{6-4-\underline{5}-3-2-1\}$ & 610 \\
5 & $\{2-\underline{-}-1-6-4-3\}$ & 626 & 5 & $\{6-\underline{5}-4-3-2-1\}$ & 612 \\
6 & $\{\underline{5}-2-1-6-4-3\}$ & 672 & 6 & $\{\underline{5}-6-4-3-2-1\}$ & 633 \\
\hline
\end{tabular}

${ }^{*}$ Best solution among all candidate solutions in this step.

${ }^{\ddagger}$ Three different solutions have the same minimum makespan.

Step 2. All candidate solutions are listed in Table 11.

Step 3. $S^{1}:=\{2-1-6-4-3-5\}$, which is the best solution, and $S^{2}:=\{6-4-3-2-1-5\}$, which is the other best solution (when there are multiple best solutions, we arbitrarily designate one of the multiple best solutions as $S^{1}$ and another best solution as $S^{2}$, resp.). $U:=\{5\} . x:=6$. Go to Step 1 .

Step $1(U \neq \emptyset)$. Thus, select $S_{6}$, that is, job 5 .

Step 2. All candidate solutions are listed in Table 12.

Step 3. $S^{1}:=\{2-1-6-4-3-5\}$, which is the best solution, and $S^{2}:=\{6-4-3-2-1-5\}$, which is the other best solution. $U:=\{\}$. $x:=6$. Go to Step 1 .
Step $1(U=\emptyset)$. Thus, go to Step 4 .

Step 4. $S^{1}$, that is, $\{2-1-6-4-3-5\}$, is the final solution. STOP.

The final solution of BS(2) for the example problem has the makespan of 571, which has been improved from the initial solution obtained by JS, whose makespan was 623 .

\section{Notations}

Parameters

$N$ : Number of jobs

$R: \quad$ Number of machines at Stage 2

$p_{i k}$ : Processing time of job $i$ at Stage $k$

$s_{i j}$ : Sequence-dependent setup time between jobs $i$ and $j$ at Stage 1 .

Variables

$c_{i k}$ : Completion time of job $i$ at Stage $k$

$x_{i j k}: 1$, if job $i$ is sequenced immediately before job $j$ on the same machine at Stage $k ; 0$, otherwise

$z$ : Makespan, that is, the completion time of the job finished last at Stage 2.

\section{Conflict of Interests}

The authors declare that there is no conflict of interests regarding the publication of this paper.

\section{Acknowledgment}

This paper was supported by Konkuk University in 2013.

\section{References}

[1] R. Ruiz and J. A. Vázquez-Rodríguez, "The hybrid flow shop scheduling problem," European Journal of Operational Research, vol. 205, no. 1, pp. 1-18, 2010.

[2] H. S. Mirsanei, M. Zandieh, M. J. Moayed, and M. R. Khabbazi, "A simulated annealing algorithm approach to hybrid flow shop scheduling with sequence-dependent setup times," Journal of Intelligent Manufacturing, vol. 22, no. 6, pp. 965-978, 2011.

[3] H.-T. Lin and C.-J. Liao, "A case study in a two-stage hybrid flow shop with setup time and dedicated machines," International Journal of Production Economics, vol. 86, no. 2, pp. 133-143, 2003.

[4] A. Rossi, A. Puppato, and M. Lanzetta, "Heuristics for scheduling a two-stage hybrid flow shop with parallel batching machines: application at a hospital sterilisation plant," International Journal of Production Research, vol. 51, no. 8, pp. 23632376, 2013.

[5] J. N. D. Gupta, "Two-stage, hybrid flowshop scheduling problem," Journal of the Operational Research Society, vol. 39, no. 4, pp. 359-364, 1988.

[6] M. E. Kurz and R. G. Askin, "Scheduling flexible flow lines with sequence-dependent setup times," European Journal of Operational Research, vol. 159, no. 1, pp. 66-82, 2004. 
[7] M. Zandieh, S. M. Fatemi Ghomi, and S. M. Moattar Husseini, "An immune algorithm approach to hybrid flow shops scheduling with sequence-dependent setup times," Applied Mathematics and Computation, vol. 180, no. 1, pp. 111-127, 2006.

[8] B. Naderi, M. Zandieh, and V. Roshanaei, "Scheduling hybrid flowshops with sequence dependent setup times to minimize makespan and maximum tardiness," International Journal of Advanced Manufacturing Technology, vol. 41, no. 11-12, pp. 11861198, 2009.

[9] T.-H. Park, H.-J. Kim, and N. Kim, "Path planning of automated optical inspection machines for PCB assembly systems," International Journal of Control, Automation and Systems, vol. 4, no. 1, pp. 96-104, 2006.

[10] X. Zhu and W. E. Wilhelm, "Scheduling and lot sizing with sequence-dependent setup: a literature review," IIE Transactions, vol. 38, no. 11, pp. 987-1007, 2006.

[11] P. S. Ow and T. E. Morton, "Filtered beam search in scheduling," International Journal of Production Research, vol. 26, no. 1, pp. 35-62, 1988.

[12] M. Nawaz, E. E. Enscore Jr., and I. Ham, "A heuristic algorithm for the $m$-machine, $n$-job flow-shop sequencing problem," Omega, vol. 11, no. 1, pp. 91-95, 1983.

[13] G.-C. Lee, S. Choi, D. Hong, and S.-H. Choi, "Heuristic algorithms for the path planning problem in three-dimensional automated optical inspection (AOI) machines," International Journal of Control and Automation, vol. 6, no. 4, pp. 321-328, 2013.

[14] S. M. Johnson, "Optimal two- and three-stage production schedules with setup times included," Naval Research Logistics Quarterly, vol. 1, no. 1, pp. 61-68, 1954.

[15] T. C. E. Cheng and B. M. T. Lin, "Johnson's rule, composite jobs and the relocation problem," European Journal of Operational Research, vol. 192, no. 3, pp. 1008-1013, 2009.

[16] Q.-K. Pan, L. Wang, and B.-H. Zhao, "An improved iterated greedy algorithm for the no-wait flow shop scheduling problem with makespan criterion," International Journal of Advanced Manufacturing Technology, vol. 38, no. 7-8, pp. 778-786, 2008.

[17] S. Kirkpatrick, J. Gelatt, and M. P. Vecchi, "Optimization by simulated annealing," Science, vol. 220, no. 4598, pp. 671-680, 1983.

[18] D. S. Johnson, C. R. Aragon, L. A. McGeoch, and C. Schevon, "Optimization by simulated annealing: an experimental evaluation; Part I, graph partitioning," Operations Research, vol. 37, no. 6, pp. 865-892, 1989. 


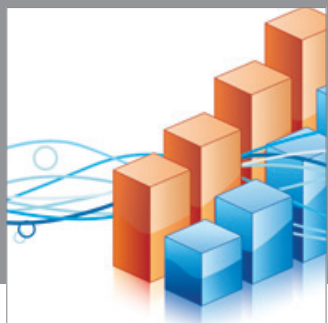

Advances in

Operations Research

mansans

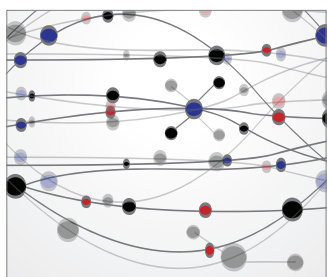

The Scientific World Journal
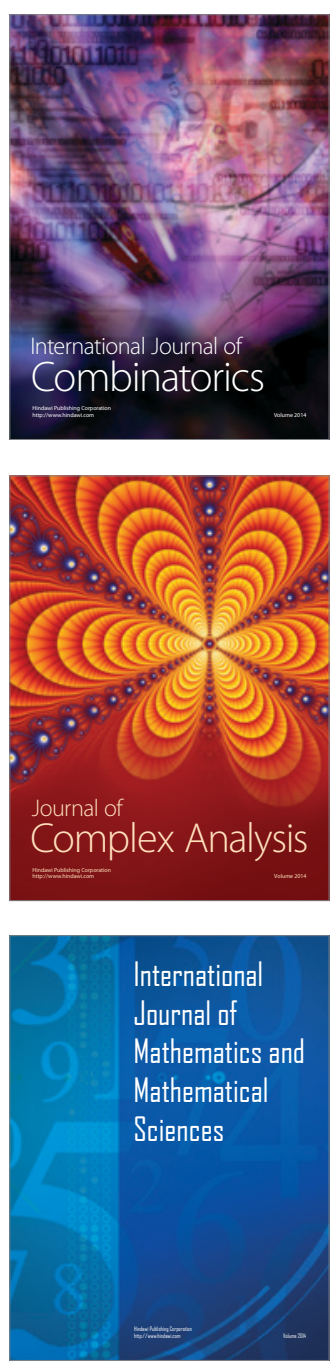
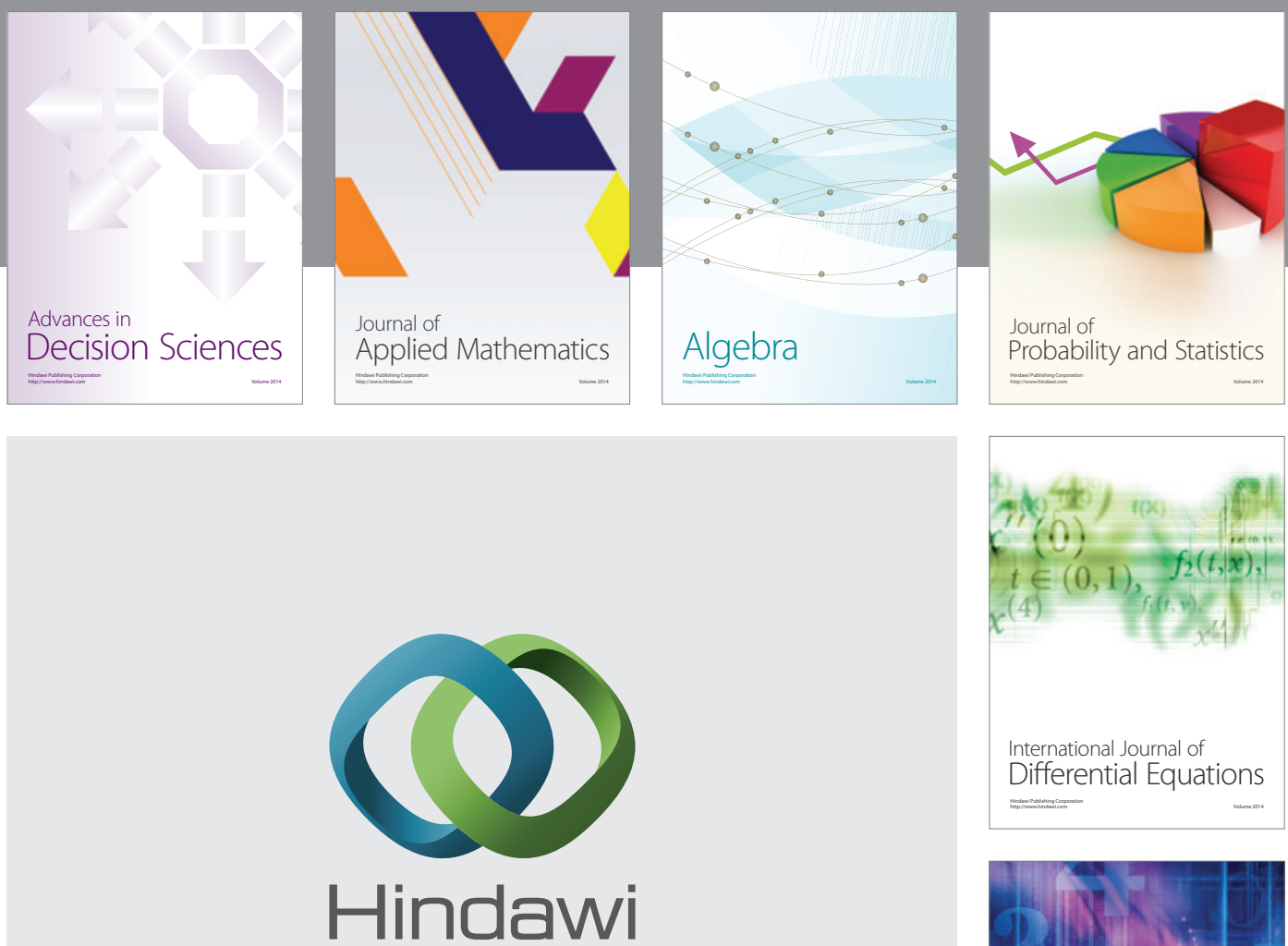

Submit your manuscripts at http://www.hindawi.com
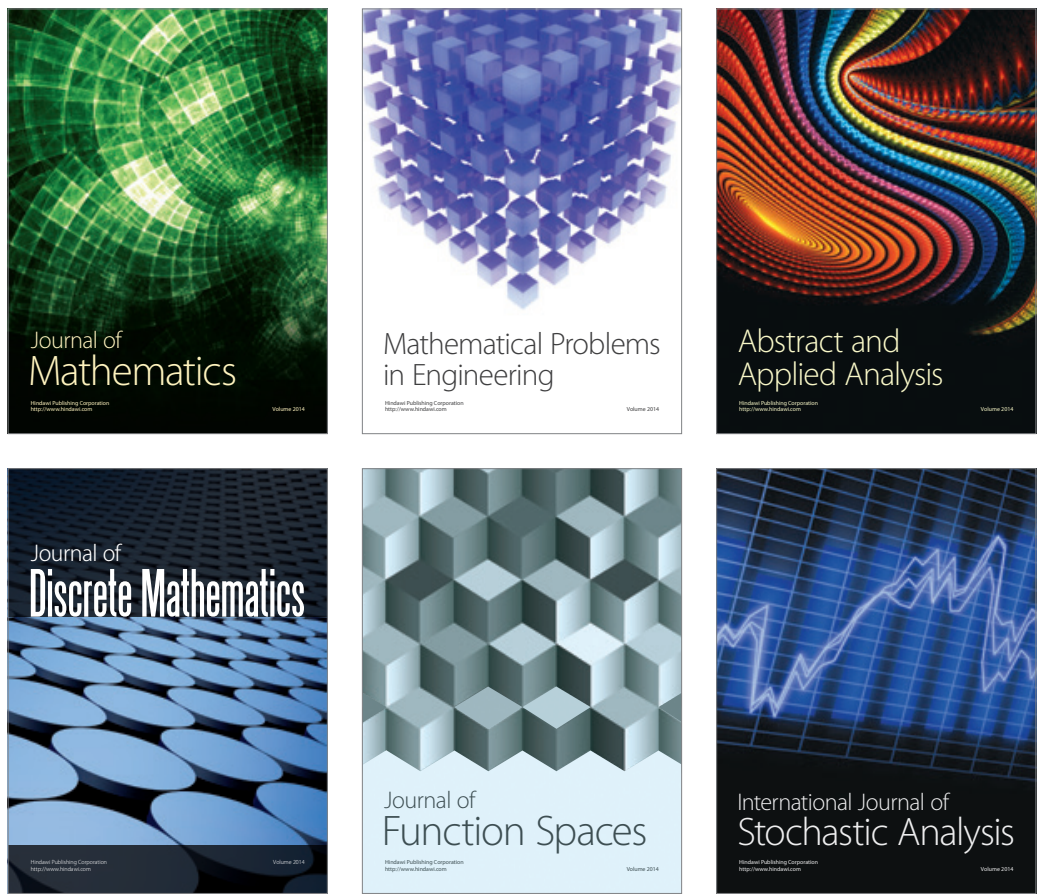

Journal of

Function Spaces

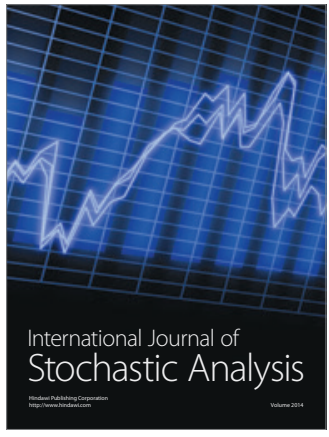

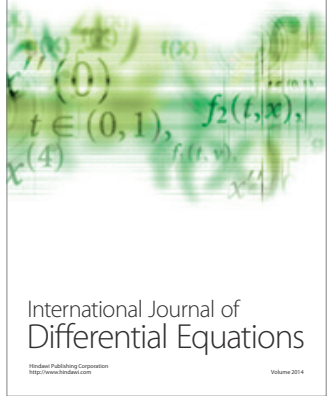
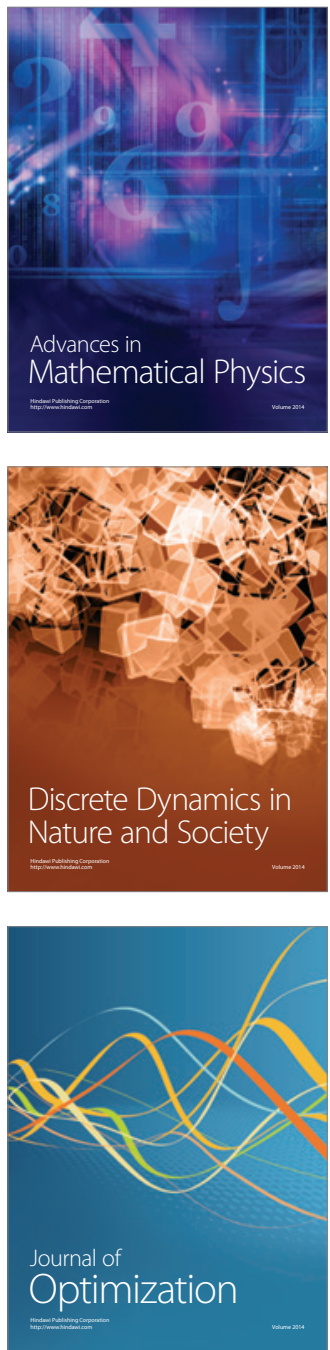